$56^{\text {ème }}$ Congrès de la SFMBCB, 02016 (2011)

DOI: $10.1051 / \mathrm{sfmbcb} / 20115602016$

(C) Owned by the authors, published by EDP Sciences, 2011

\title{
Métastase mandibulaire d'un ostéosarcome multicentrique. Présentation d'un cas clinique
}

\author{
El Harti $K^{1}$, Bennani $A^{1}$, Oujilal $A^{2}$, El Wady $W^{1}$ \\ ${ }^{1}$ Faculté de Médecine dentaire, Université Mohamed 5 Souissi, Rabat, Maroc \\ ${ }^{2}$ Service d'ORL et de Chirurgie cervico-faciale, Hôpital des Spécialités, CH Ibn Sina, Rabat, \\ Maroc \\ karimaelharti@yahoo.fr
}

L'ostéosarcome (OS) est une tumeur conjonctive maligne productrice d'une matrice ostéoïde. Il représente environ $30 \%$ des tumeurs osseuses malignes primaires et touche généralement les patients ayant entre 10 et 30 ans. Les métaphyses des os longs sont les plus fréquemment intéressées, en particulier la portion distale du fémur et proximale du tibia (Nakamura et al. 2001). La majorité des métastases des OS des os longs intéresse les poumons, puis les os (10\%) (Pakos et al. 2009). Cependant, les métastases des OS dans les maxillaires sont extrêmement rares.

Ce travail rapporte le cas d'une métastase mandibulaire extrêmement rare d'un OS multicentrique dont la tumeur primitive se situe sur le plateau tibial gauche. Une femme, âgée de 23 ans a consulté pour une tuméfaction mandibulaire gauche présente depuis 25 jours. Seize mois auparavant, elle avait subi un traitement chirurgical, une chimiothérapie et une radiothérapie pour un OS de la jambe gauche. L'examen exobuccal a révélé une adénopathie sous-maxillaire gauche, dure, douloureuse et fixée au plan profond. L'examen endobuccal a révélé une lésion mandibulaire bilobée, entre 33 et 37, de couleur violacée, ferme et douloureuse à la palpation. Les dents en rapport avec la tumeur étaient mobiles. L'orthopantomogramme a montré une image lacunaire diffuse entraînant une alvéolyse des dents bordant la lésion, la TDM une image hypodense parasmphysaire gauche, bien limitée, homogène, mesurant $35 \times 30 \mathrm{~mm}$. La biopsie a montré une lésion constituée de cellules géantes. Le traitement a donc consisté en une simple énucléation. La lésion a récidivé un mois plus tard ; une hémimandibulectomie et un curage ganglionnaire sous-maxillaire gauche ont été réalisés. L'examen anatomopathologique a conclu à une métastase d'un OS ; le parenchyme ganglionnaire était dépourvu d'éléments tumoraux. La scintigraphie osseuse à l'HMDP-Tc $99 \mathrm{~m}$ a montré de nombreux foyers d'hyperfixation intéressant le crâne, les épaules, les côtes, le rachis, le bassin et les fémurs et a conclu à une ostésarcomatose. Une chimiothérapie a été indiquée pour le traitement des autres localisations secondaires de l'OS.

Seulement neuf cas d'OS mandibulaires secondaires ont été rapportés dans la littérature (Cataldo et al. 1965, Snyder et al. 1968, Ohba et al. 1975, Stroncek et al. 1981, Suzuki et al. 1999, Nakamura et al. 2001, Daffner et al. 2002, Behere et al. 2009). La métastase mandibulaire des OS peut être osseuse ou gingivale (Suzuki et al. 1999). Les OS peuvent être multicentriques et sont actuellement considérés comme une pathologie métastatique (Daffer 1997). Deux formes sont alors distinguées : la forme synchrone avec plusieurs foyers tumoraux dans les premiers 5 mois suivant la tumeur primitive et la forme métachrone associant des foyers multiples apparaissant 5 mois après la tumeur primitive. La survenue de métastases squelettiques sans métastases pulmonaires se fait à travers le système veineux vertébral (Hopper 1990). Le traitement chirurgical des OS primaires maxillo-faciaux permet une survie à long terme des patients. Cependant, le traitement des métastases buccales n'est pas codifié en raison de sa faible incidence. La chimiothérapie a permis d'augmenter le taux de survie des patients de $20 \%$ dans les années 1960 à 70\% dans les années 1980 (Fernandes et al. 2007). 